\title{
From hybrids to hermaphrodites in population genetics
}

\author{
Robert Verity ${ }^{*+1}$, Jeannine Marquardt ${ }^{+1,2}$, Andrea Hatlen $^{\dagger 1}$ and Jasmin Zohren ${ }^{+1}$
}

\begin{abstract}
A report on the 46th annual PopGroup conference, Glasgow, UK, December 18-21, 2012.

Keywords Genetics, evolution, coalescent theory, selective sweeps, blogging, gynodioecy, androdioecy
\end{abstract}

Shortly before Christmas 2012, the 46th PopGroup (Population Genetics Group) meeting was held at Glasgow University, UK. Over 180 scientists attended from 19 different countries, with speakers from diverse research areas and ranging from $\mathrm{PhD}$ students to retired professors. Some talks dealt with the conservation of exotic species on remote islands, while others took a more theoretical approach. Almost all made use of, or anticipated, the volume and ever-reducing cost of data from nextgeneration sequencing, which is opening up access to research topics and study organisms that were previously off limits. Here, we report on some of the main themes of the conference, and the directions in which nextgeneration sequencing technology is taking us.

\section{Hybridization in non-model organisms}

In the opening plenary talk, Michael Arnold (University of Georgia, Athens, USA) recounted how his long-term population genetic research on hybrids of the Louisiana Iris has unfolded. An apparently disastrous flood changed the direction of one of Arnold's large-scale field experiments, providing evidence that the proportion of introgressed genes from one parental species dictated how well individuals survived flooding. A recurring theme of the conference, particularly in the study of genetic introgression, was that dissection of such effects in non-model organisms in their natural environment was most

\footnotetext{
${ }^{\dagger}$ All authors contributed equally to this work

*Correspondence: r.verity@qmul.ac.uk

'School of Biological and Chemical Sciences, Queen Mary University of London,

Mile End Road, London, E1 4NS, UK

Full list of author information is available at the end of the article
}

effective through the combination of new sequencing technologies with field surveys, experimentation and hard-won biological insights.

This combination was exemplified by the work on hybridization between two subspecies of the grasshopper Chorthippus parallelus presented by James Hutchison (University of Sheffield, UK). Whereas laboratory crosses between the two taxa produce sterile males, the offspring produced in a narrow hybrid zone in the Pyrenees mountain range seem to be fully fertile. This result could be explained by thousands of years of selection, which might, for example, drive key sterility alleles out of the zone. But how could the key loci be identified from the many differences between the taxa across the whole genome? Hutchison evaluated candidate loci from expression data by analyzing allele frequency differences across the zone. This strategy exploits the long history of crossing, backcrossing, gene flow and selection producing the frequency differences.

In the case of trees, a long temporal perspective is provided by the living individuals themselves. Kirsten Wolff (Newcastle University, UK) presented preliminary results on hybridization in lime trees. Ancestors, perhaps thousands of years old, live alongside their descendants, making them living fossil records and facilitating direct genetic comparisons over these extraordinary timescales. On the other hand, the existence of overlapping generations with their genetic contribution to new offspring presents an analytical challenge in the form of incomplete lineage sorting.

\section{Theoretical surprises}

The wealth of data provided by next-generation sequencing methods brings with it the statistical power to detect evolutionary change at a high resolution. Theoretical models play an important role here, separating evolutionary signal from noise and providing tentative explanations for differences throughout the genome. This year's theoretical talks put forward some novel and unexpected predictions that in some cases went so far as to defy the conventional wisdom. Daniel Weissman (Institute of Science and Technology, Klosterneuberg, Austria) gave a prize-winning talk about the counterintuitive effects of 
selective sweeps. Using both analytical theory and simulation, Weissman demonstrated that the random origin of advantageous mutations in a two-dimensional bounded population systematically distorts the pattern of ancestry. These mutations seed selective sweeps spreading out to the species' margins, such that the ancestry of the adjacent part of the genome will trace back toward the center of the species' range, resulting in a pattern of neutral variation that could be easily mistaken for a range expansion.

In the case of genuine range expansions, Jens Nullmeier (Max Planck Institute for Dynamics and Self-Organization, Göttingen, Germany) argued for a clear distinction between phenotype-limited range expansions and those in which the population is limited by a slowly moving boundary. Nullmeier developed a coalescent model, and concluded that the different types of range expansions leave different genetic footprints, which can potentially be used to infer past population histories.

Another prize-winning talk exploited genome-wide data to explore some challenges inherent in estimating species divergence times from very large numbers of genes. Richard Nichols (Queen Mary University of London, UK) analyzed a dataset of thousands of genes across vertebrate genomes, and discovered that estimates of the time to the last common ancestor of humans and chimpanzees vary greatly between human chromosomes. Yet most discrepancies could be resolved when certain multi-gene families were removed from the analysis, suggesting that gene conversion might be at play in driving this phenomenon. Following up with a theoretical model, Nichols demonstrated that gene conversion accelerates the rate at which species differentiate, particularly in the period immediately after speciation. If ignored, this effect could grossly distort estimates of divergence time or effective population size for surrounding parts of the genome.

Genome-wide data can also be used to characterize allele frequencies at a large number of loci within the same species, as demonstrated by Brian Charlesworth (University of Edinburgh, UK), who presented work exploring the effects of mutational bias on stabilizing selection. Most standard evolutionary models predict that selection is more effective in large populations than in small populations as a result of weaker genetic drift. However, by introducing mutational bias into a model of stabilizing selection, the scaled intensity of selection is nearly independent of effective population size over a wide range of parameter space; if this model holds true, then it may be impossible to distinguish between stabilizing and directional selection from allele frequency data alone.

\section{A role for sex?}

Another challenge of moving from the laboratory into the natural world is that present-day biology will differ from that which shaped the evolution of the genome. Even attributes as fundamental as breeding systems are labile: Deborah Charlesworth (University of Edinburgh, UK) presented work on the breakdown of dioecy (separate sexes) to gynodioecy (females and hermaphrodites) in angiosperms. A dioecious population evolves to the gynodioecious form of sexuality when males become hermaphrodites by acquiring some female attributes. The study modeled the common gynodioecious situation and found that the frequency of females can be higher than hermaphrodites if the females outnumber the males in the ancestral dioecious population.

Sonia Consuegra (Aberystwyth University, UK) explored the reasons why the fish Kryptolebias marmoratus might maintain some sexuality in an androdioecious population with many selfing hermaphrodite lineages. Consuegra's group found that, given a choice, males exhibited a mating preference for the more genetically different mate, although, intriguingly, the hermaphrodites did not. The observation of higher parasite levels in individuals derived from self-fertilization may explain the continued existence of males in this system.

Heidi Aisala (University of Oulu, Finland) examined the balance of cloning and sexual reproduction in fish parasites. Parasites from the genus Gyrodactylus are predominantly host-specific, but with occasional sexual reproduction they can gain the capacity to switch host species. Thereafter they can continue their clonal reproduction, needing only one individual to start the new population. This strategy has proved highly successful, to the extent that Gyrodactylus host-switching is becoming a major economic and ecological problem. The movement of infected individuals by fish farmers has allowed the parasite to invade local waterways and jump to new fish species.

\section{Opening up the scientific process}

Probably one of the most controversial talks at the conference was the final plenary talk, entitled 'Why I blog instead of writing papers', by Roderic Page (University of Glasgow, UK). A few years ago, Page shifted the balance of his publication toward blogging and away from writing academic papers. In a spirited debate, the audience raised concerns that this method of communication is more self-centered than conventional publishing. However, Page insisted that the use of blogging encourages feedback and ensures that science is a two-way discussion, as it should be. Page looked forward to a time when DOIs (digital object identifiers, such as those used for online articles in academic journals) are made available for blog posts, thereby making them more readily citable. He argued that these hoped for citable blog posts will be the future mode of communicating science. There are clearly still open questions as to how this scenario would work 
with regard to peer review and ownership of results, but Page nevertheless predicts that the worlds of blogging and academic publishing will begin to merge.

\section{Conclusions}

The technological advances of the last few years are changing the way we do and communicate science. Nextgeneration sequencing technologies allow for large scale and high-resolution analyses of individuals and populations, revealing surprising patterns that feed directly back into the theoretical models. The way in which we communicate results is also being altered by blogging and other social media, and is forcing us to rethink the way we interact with the scientific community, as well as with the population at large. All in all, this is an exciting time in population genetics.

\section{Abbreviations}

DOI, digital object identifier.

Competing interests

The authors declare that they have no competing interests.

\section{Acknowledgements}

We thank the conference organizers, especially Barbara Mable, the Marie-Curie Initial Training Network INTERCROSSING for funding the authors, and Richard Nichols for comments.

\section{Author details}

'School of Biological and Chemical Sciences, Queen Mary University of London, Mile End Road, London, E1 4NS, UK. 2Life Sciences, Natural History Museum London, Cromwell Road, London, SW7 5BD, UK.

Published: 28 January 2013

doi:10.1186/gb-2013-14-1-301

Cite this article as: Verity $R$, et al.: From hybrids to hermaphrodites in population genetics. Genome Biology 2013, 14:301. 